\title{
CHARACTERISTIC FEATURES OF MULTIPLE ORGAN FAILURE IN CASES OF PERITONEAL SEPSIS
}

\author{
L. Yu. Ivashchuk, I. B. Pizhitsky \\ I. HORBACHEVSKY TERNOPIL STATE MEDICAL UNIVERSITY, TERNOPIL, UKRAINE
}

Background. The study of hystomorphology of liver and small intestine in experimental peritonitis is presented. Due to this information the criterion of pathogenetic moment transition SIRS for peritoneal sepsis was determined.

Objective. The aim of the research was to study the morphology of terminal part of small intestine and liver in cases of experimental peritonitis.

Methods. For histological and electron microscope study the biopsy of liver, small intestine were taken; the samples were stabilized in a neutral formalin, dried in alcohol of increasing concentration and placed in paraffin. Paraffin sections were painted with hematoxylin and eosin and studied under the light-optical microscope.

Results. Apoptosis caused damage to enterocytes and hepatocytes of first bacterial translocation. Mechanism of vasodilatation effect of NO and its effect on apoptosis were determined. Septic shock was accompaniment of two main levels of body cells damage: apoptosis and membranes destruction. Peritoneal sepsis is a grave condition caused by progressive peritonitis and polyorgan insufficiency syndrome. The phases of peritonealis sepsis pathogenesis were defined.

Conclusions. The presented morphological criteria prove the initiation of apoptosis within 24 hours after the development of peritonitis in enterocytes and hepatocytes.

KEY WORDS: peritonealis sepsis; apoptosis; polyorgan insufficiency syndrome.

\section{Introduction}

Twenty-five years ago at the Chicago Consensus Conference on Sepsis some traditional approaches and postulates as well as critical approach to the suggested concept has been changed $[1,2]$.

The accumulation of experimental and clinical data on the significance of cytokines in the pathogenesis of sepsis allows adequate formulation of clinical diagnosis and formation of accurate subject $[5,7]$.

The realization of systemic inflammatory response syndrome (SIRS), as a part of diagnosis that characterizes the septic state of patients, allowed building algorithm due to pathogenic effects.

At the same time, much criticism by the supporters of the traditional concept of 'sepsis as a bacteremia accompanied by appropriate clinical disruption of homeostasis' that are inherent to SIRS substantiate that clinical signs

Corresponding author: Larysa Ivashchuk, Department of General Surgery, I. Horbachevsky Ternopil State Medical University, 2 Shpytalna street, Ternopil, Ukraine, 46008

Phone number: +38679401031

E-mail: ivashchuk_lu@tdmu.edu.ua of the syndrome are a common reaction to surgical infection.

In our clinic, the researchers believe that the determination of septic states is not reasonable enough: sepsis, severe sepsis, septic shock, hypotension syndrome and multiple organ failure.

The concept peritoneal (abdominal) sepsis is even more terminologically confusing.

Our aim was to define the morphological criteria, which determine pathogenic moment of SIRS transition that causes peritoneal sepsis at peritonitis.

\section{Methods}

During the research the morphology of terminal part of small intestine and liver in experimental peritonitis was studied.

The research took place in spring. 20 mature nonlinear white male rats, weighing $200-250 \mathrm{~g}$ were used. The animals were kept on a standard vivarium diet with free access to water in accordance with the requirements of the "European Convention for the Protection of Vertebrate Animals used for Experimental and Other Scientific Purposes" [6, 9]. 
Acute peritonitis was modeled by administration of $10 \%$ fecal mixture into abdominal cavity by the method of Lazarenko V. A., et al (2008) [8] that is comparable to etiological factors, clinical manifestations and phase transition of similar process in humans and allows the death of animals, which is acceptable for conducting a dynamic study during 10 days. This was performed by the administration of $0.5 \mathrm{ml}$ of $10 \%$ fecal suspension filtered into peritoneal cavity of the studied rats. The suspension was obtained by mixing isotonic solutions and feces from the intestine of two or three intact animals and then it was filtered twice through a double layer of gauze. The resulting suspension no later than in 20 minutes after preparation was administered to the intact animals by puncture method. To avoid damage to internal organs when the fecal suspension was introduced into abdominal cavity, the animals were held upright, caudal end up. By the method of puncture of ventral wall in the middle of the central line of abdomen, the end of the needle was rotated in turn into the right and left hypochondria, the right and left ilium sections, and the same amount of fecal suspension was injected. In 24 hours the animals were killed by decapitation under general anaesthesia.

For histological and electron microscope study biopsies of liver, small intestine were taken. The samples were stabilized in neutral formalin, dried in alcohols of increasing concentration and placed in paraffin. Paraffin sections were painted with hematoxylin and eosin and studied under the light-optical microscope.

Electron microscope examination of biopsy samples was processed by standard methods and placed in epoxy resin. In addition, the method was used to identify membrane permeability due to $\mathrm{Ca}^{++}$-ATP. Thick ultrathin sections $600 \AA$ were managed in ultra-microtome LKB and Reyhert. To improve the contrast the sections were painted by Reynholds and studied through the electron microscope EM-400, Philips.

\section{Results}

Histological examination of small intestine wall in all animals, which were simulated with diffuse peritonitis, proved dystrophic and destructive changes of all its layers, mainly affecting submucosal tissues: oedema on the background of disturbed intra-organ blood flow was the most pronounced that was manifested with plethora expansion and main mass of micro- vessels, perivascular and interstitial haemorrhage. Associated with oedema, histo-architectonic connective tissue stroma was damaged. Mucous membrane was sometimes flaky in intestine and a large mass of microvilli was swollen and deformed. Epithelial vessels microvilli were swollen with a clarified cytoplasm and basophilic nucleus was eccentrically placed. Muscle shell was also swollen with more extended and filled with blood microvessels, as a result focal violations moved muscle fibres with expansion to intramuscular spaces.

The electron microscope study of columnar epithelium intestinal mucosa proved pronounced dystrophic and destructive changes of nucleus and cytoplasm. Nuclei fracture, chromatin aggregation and redistribution of its overwhelming pre-shell condensation were established; normal chromatin in the nuclei of epithelial cells of intestine was placed uniformly in plasma nuclei.

In the cytoplasm of enterocytes intracellular accumulation of detritus was evidenced, proving fairly pronounced destructive changes in these cells. Changes occur in the microvilli system that protrude the intestine in a gleam. Histochemical reaction on membrane permeability using the $\mathrm{Ca}^{++}$-ATP-ase proved that compare to normal, reaction products fell in the sediment on the surface membranes of microvilli and determined in their gleam penetrating into the cytoplasm of cells. This evidenced membrane permeability microvilli violation associated with their swelling.

External intestinal serosa studied on lightoptical level was slightly thickened and swollen. Violations of connective tissue skeleton swelling and disorientation of collagen and elastic fibers and desquamation mesothelial cells were also noticed. Numerous cavities extended microvascular events with accumulation of red blood cells and endothelial swelling. In the parietal layer of peritoneum the changes were less pronounced and comprised extended microvessels cavity and perivascular connective tissue swelling (Fig. 1.)

This resulted in pathological permeability of intestine inner lining and was accompanied by increased penetration of endotoxin, bacterial contamination from intestine cavity into mesenteric lymph nodes and portal system.

Microscopic examination of liver in all cases proved more or less pronounced swelling of parenchyma on the background of disturbed intra-organ blood flow, which was manifested by plethora expansion and main vessels mass- 


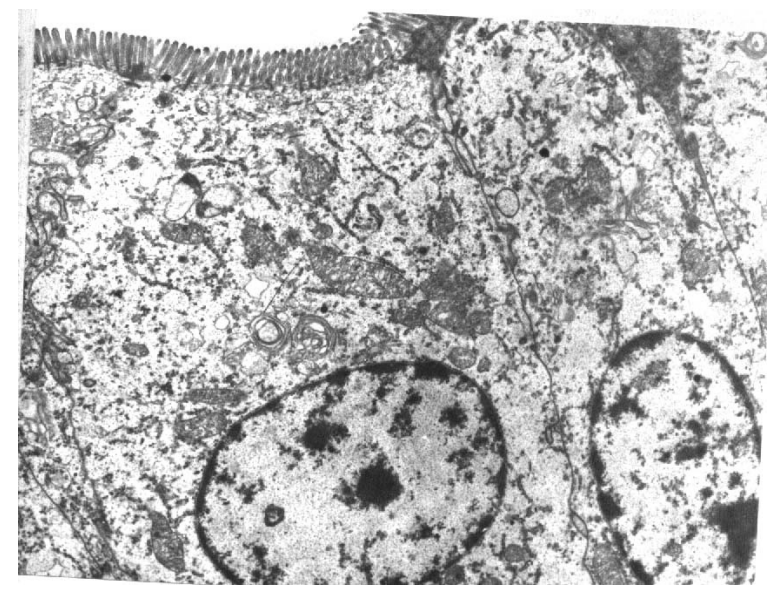

Fig. 1. Modeling of acute peritonitis. Oedema and violation of intracellular organelles integrity of intestine epithelial cells. Electron micrographs $\times 13000$

es and focal perivascular haemorrhage. In areas of disturbed intra-organ blood flow quite pronounced swelling of liver cells with impaired liver histological structure and expanding intercellular spaces was evidenced. The investigation of hepatocytes structure in a significant increase in a series of semi-thin slices proved pronounced degenerative changes of liver cells with sharp cytoplasm enlightenment, significant decrease in the size of the nucleus, associated with swelling, decrease in the number and pre-shell condensation of chromatin. In addition, significantly increased sinusoids with focal desquamation of endothelial cells that route to the cavity of blood vessels was observed.

Electron microscopic examination of hepatocytes proved that some of these cells had pronounced degenerative changes associated with swelling of cytoplasm, manifested by quite significant expanding and vacuolization of endoplasmic reticulum, mitochondria and destruction of the significant decrease in the number of free and fixed ribonucleic granules. Ultrastructural changes were detected in endothelial cells of hepatic sinusoid. These changes were of diverse nature: from dystrophic-atrophic to hyperplastic, proving the focal reparative effects. In hepatocytes that were adjacent to hyperplastic endothelial cells the effects reparative regeneration were established that comprised strengthening their energy-producing and protein synthesis function by hypertrophy of mitochondria and increase in the number of free ribosomes and polysomes (Fig. 2.).

Thus, the presented morphological criteria proved the initiation of apoptosis in enterocytes and hepatocytes within 24 hours after the development of peritonitis.

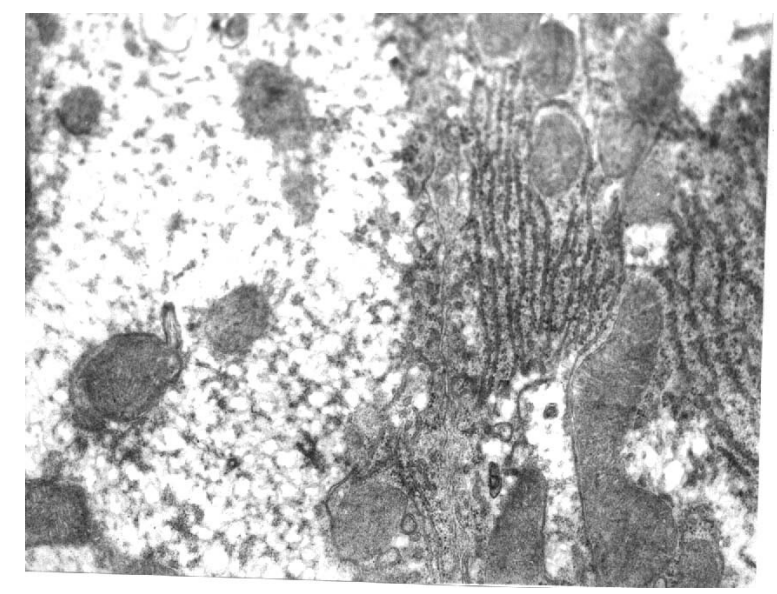

Fig. 2. Modeling of acute peritonitis. Oedema of hepatocyte with violation of mitochondria integrity and reduction of endoplasmic reticulum. Electron micrographs $\times 13000$

\section{Discussion}

Kerr [2] described these effects in 1972 as 'apoptosis' cause, we believe that further destruction of cellular organelles took place. Thus, in most enterocytes the damaged mitochondria, swollen and infected reticulum cisterns with a reduced number of ribonucleic granules fixed in their membrane were evidenced. The number of ribosomes and polysomes was reduces.

According to the literature, the mentioned processes cause local activation of immune cells, cytokines and other inflammatory mediators (TNF, IL-1,6,8), which are able to stimulate the production of prostaglandins, free radicals, nitric oxide, which in turn is a powerful factor in adaptive processes at a cellular level $[10,11]$.

The destruction of liver cells during peritonitis occurs also due to apoptosis. It is likely that this process is initiated by nitric oxide, which in living organisms is formed by the action of specific enzymes united under the title of nitric oxide synthase. The last were found in endothelial cells of arteries and veins of intestine, and were initiated by the generation of nitric oxide in all cells of liver $[3,4]$.

These changes may be enough to form multiple organ failure, especially such as bacterial translocation. In our opinion, the appearance of morphological, biochemical and clinical signs of organ failure should be the criteria for diagnosis 'peritoneal sepsis' (Fig. 3.). Further development of the pathological process is determined by two-way cell destruction: by apoptosis and degradation of membranes, and can lead to septic shock. If at this moment intensive multicomponent therapy does not help stabilize homeostasis, the irreversible changes 


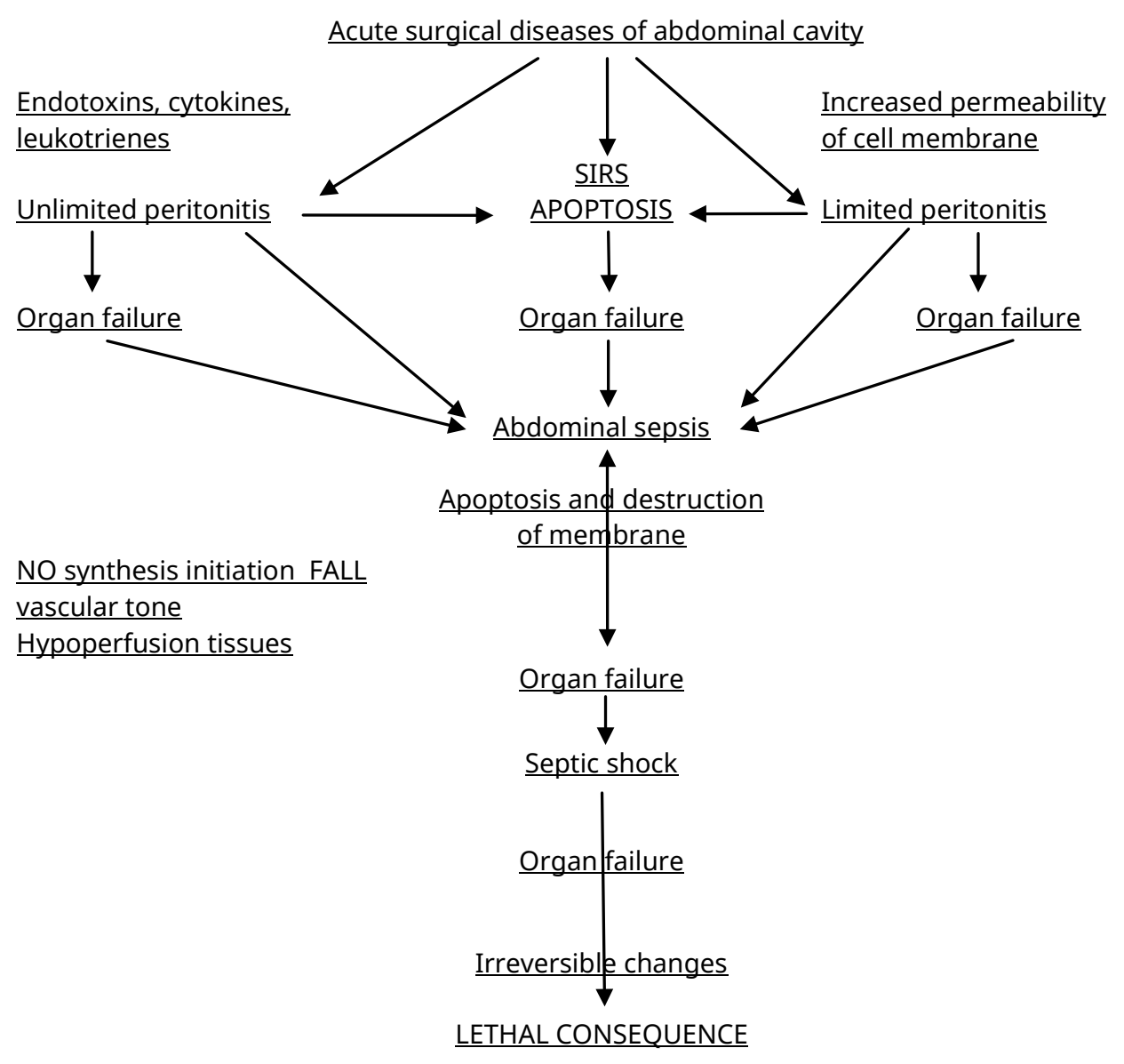

Fig. 3. Pathogenetic variants of peritoneal sepsis

in vital organs and systems will take place that may lead to fatal consequences.

We understand that the above concept is hypothetical and disputable to some extent, but morphological changes prove the need for further study of the problem, especially the determination of nitric oxide, its synthase and apoptosis.

\section{Conclusions}

The affection of hepatocytes and enterocytes in experimental peritonitis begins with apoptosis.

Apoptosis, probably, can be morphological criteria of multiple organ failure initiation.

Peritoneal sepsis is a severe pathology resulting in progression of peritonitis and multiple organ failure.

\section{References}

1. Bele R. Pathophysiology of septic shock. Congress of anaesthesiology. Jerusalem. Israel. October 2-7. 1994;140-145.

2. Boun R. Sepsis and septic shock. Congress of anaesthesiology. Jerusalem. Israel. October 2-7. 1994;125-139.

3. Gilbert RS, Hershman HR. J Cell Physiol. 1993;57:128-132.

4. Kerr J FR, Wylle AH, Currie AR. Br J Cancer. 1992;26:239-257.
5. Boyko VV, Kryvoruchko IL, Teslenko SI, Syvozhelezov AV. Common purulent peritonitis. Kharkiv: Prapor. 2008. p. 278.

6. Vlizlo VV, Fedoruk RS, Ratych IB. Laboratory methods in biology, animal husbandry and veterinary medicine: a guide. Lviv: SPOLOM. 2012;764.

7. Dziubanovskyi IYa, Mihenko BO. Organ failure syndrome and its correction in patients with acute peritonitis. Ukrainian Journal of Surgery. 2009; 2:56-59. 
8. Lazarenko VA, Lipatov VA, Blinkov YuYu, Skorikov DV. Experimental model of widespread fecal peritonitis. Man and his health. 2008;4:128-132.

9. Kozhemiakin YuM, Khromov OS, Filonenko MA, Saifetdinova HA. Scientific and practical advice on keeping laboratory animals and work with them. Kyiv: Avitsena. 2002;156.

10. Titov VN. The role of macrophages in the formation of inflammation, the effect of interleu- kin-1, interleukin- 6 and the activity of the hypothalamic-pituitary system. Clinical laboratory diagnostics. 2003;12:3-10.

11. Boelen A, Kwakkel J, Platvoetter Schiphorst M. Interleukin-18, a proinflammatory cytokine, contributes to the pathogenesis of non-thyroid al illness mainly via the central part of the hypothalamuspituitary-thyroid axis. Eur J Endocrinol. 2004; 151(4):497-502.

Received: 2017-06-22 